\title{
El tratamiento psicológico de los desórdenes emocionales en Atención Primaria. Análisis de un caso clínico.
}

\section{Psychological treatment of emotional disorders in Primary Care. Analysis of a clinical case.}

Fecha de recepción: 26-03-2018

Fecha de aceptación: 21-06-2018
Marina Baquero Tomás

Universidad Católica de Valencia "San Vicente Mártir". España

María Modrego Muñoz

Universidad Católica de Valencia "San Vicente Márti". España

Luciana Moretti

Universidad Nacional de Córdoba, Argentina

César González Blanch

Hospital Universitario Marqués de Valdecilla. IDIVAL. España

Itziar Iruarrizaga Díez

Universidad Complutense de Madrid. España

\section{resumen/alustract:}

La prevalencia de desórdenes emocionales (DE) ha aumentado considerablemente en los últimos años. Los servicios de Atención Primaria (AP) son los primeros receptores de la mayoría de pacientes con estas patologías, especialmente las relacionadas con la depresión, la ansiedad y las somatizaciones. En España, la proporción de estos pacientes que recibe un tratamiento mínimamente adecuado es baja, por lo que resulta necesario establecer de protocolos de tratamiento eficientes, basados en la evidencia científica, para el abordaje de los DE. El proyecto PsicAP es un protocolo de intervención grupal fundamentado en el enfoque transdiagnóstico y basado en técnicas cognitivo-conductuales. El objetivo es acercar los tratamientos psicológicos a la AP, facilitando el acceso a un porcentaje elevado de la población. A continuación, se describe el caso clínico de un paciente con un DE que participa en el proyecto PsicAP. El programa, basado en el modelo de tratamiento transdiagnóstico, se desarrolla en 7 sesiones grupales, de hora y media de duración y repartidas en 24 semanas. El programa resulta eficaz en la reducción de la sintomatología clínica, lo que confirma que se trata de un tratamiento breve, de bajo coste y útil para el abordaje de los DE en el contexto de AP.

In recent years the emotional disorders' (ED) prevalence has increased considerably. Primary Care (PC) services are the first receivers of the majority of patients with these pathologies, especially those related to depression, anxiety and somatization. In Spain, the proportion of these patients that receives a minimally adequate treatment is low. It is necessary to establish efficient treatment protocols based on scientific evidence to approach the ED. Psychology project in PC PsicAP is a groupal intervention protocol based on the transdiagnostic approach and cognitive-behavioral techniques. The objective is bringing psychological treatments closer to PC, making easier access to a high percentage of the population. Next, we describe the case of an ED patient who participates in PsicAP project. The program, based on the transdiagnostic treatment approach, is developed in 7 group sessions, with a duration of an hour and a half over 24 weeks. The program is effective in reducing clinical symptoms, which confirms that is a short, low-cost and useful treatment for ED patients in the PC context.

\section{palabras clave/keywords:}

Desórdenes emocionales, Ansiedad, Depresión, Somatizaciones, Tratamiento psicológico, Transdiagnóstico, Atención Primaria. Emotional disorders, Anxiety, Depression, Somatizations, Psychological treatment, Transdiagnostic, Primary care. 


\section{Introducción}

Los trastornos mentales son un problema creciente de salud pública, pues afectan a cientos de millones de personas y generan un enorme sufrimiento, discapacidad y cuantiosas pérdidas económicas. Recientes informes aportados por la Organización Mundial de la Salud (OMS, 2017) sugieren que se ha producido un incremento y un deterioro progresivo en los índices de salud de la población, siendo, a nivel mundial, la depresión y los trastornos de ansiedad, los más prevalentes y discapacitantes. Estudios epidemiológicos realizados en España (Haro et al., 2006) apuntan que aproximadamente una de cada cinco personas presentará un trastorno mental a lo largo de su vida, y que alrededor de una de cada diez personas, lo presentó en los últimos 12 meses. Entre los trastornos mentales más frecuentes podemos situar los denominados desórdenes emocionales (DE), que incluyen trastornos de ansiedad (trastorno de pánico, trastorno de ansiedad generalizada o trastorno por estrés postraumático), los trastornos del estado de ánimo (como el trastorno depresivo mayor o distimia) y los trastornos adaptativos (de tipo ansioso, depresivo o mixtos) (Cano-Vindel, 2011b).

Desde el Sistema Nacional de Salud (SNS), el tratamiento de estos DE se lleva a cabo, en gran medida, desde Atención Primaria (AP), pues aproximadamente dos tercios de pacientes con trastornos mentales con atendidos en este contexto. En España existe una prevalencia de trastornos mentales ligeramente menor que en otros países, pero un porcentaje mayor de atención de estos casos por el SNS, así como tasas más elevadas de hiperfrecuentación (Cano-Vindel, 2011a). El tratamiento que suele ofrecerse en AP es el tratamiento farmacológico (Kovess-Masfety et al., 2007), a pesar de que está demostrado que el tratamiento psicológico temprano mediante técnicas basadas en la evidencia científica, es el más efectivo para tratar estas patologías y prevenir el desarrollo de otros trastornos mentales, o la cronificación de los ya existentes (Bortolotti, Menchetti, Bellini, Montaguti y Berardi, 2008; Cape, Whittington, Buszewicz, Wallace y Underwood, 2010; Richards y Suckling, 2009).

Para el abordaje de los DE, los tratamientos transdiagnósticos han demostrado ser eficaces (Newby, McKinnon, Kuyken, Gilbody y Dalgleish, 2015). Asimismo, las terapias psicológicas grupales llevadas a cabo en el contexto de AP también han demostrado ser efectivas en la disminución de la sintomatología clínica, comparadas con el tratamiento habitual (Huntley, Arya y Salisbury, 2012). En los últimos años ha aumentado el número de estudios científicos acerca de la evaluación de programas basados en el tratamiento transdiagnóstico en el abordaje de los DE, llevados a cabo en diferentes países como España, Estados Unidos, Australia, o Japón (Bullis et al., 2015; Chu et al., 2016; Espejo et al., 2016; Farchione et al., 2012; García-Escalera, Chorot, Valiente, Reales y Sandín, 2016; Ito et al., 2016; Mullin et al., 2015; Osma, Castellano, Crespo y García-Palacios, 2015). Los resultados derivados de estas investigaciones revelan una reducción significativa de la sintomatología asociada a los DE una vez finalizado el tratamiento, así como un mantenimiento de estos resultados en las posteriores evaluaciones de seguimiento.

A continuación se describe el caso de un paciente que acude a su centro de AP de referencia, situado en Valencia, y es remitido al programa de tratamiento psicológico en AP correspondiente al proyecto PsicAP (Psicología en Atención Primaria). De los diferentes grupos 
de tratamiento que se han realizado en este proyecto, se ha seleccionado a un paciente de manera aleatoria de uno de los grupos de tratamiento psicológico de Valencia, mediante una aleatorización simple al azar. Este proceso se realizó sacando una papeleta de una caja con 8 papeletas, cada una con un número. Se asignó un número a cada paciente y la primera papeleta que se sacó, fue la del paciente seleccionado.

El proyecto PsicAP tiene como objetivo principal desarrollar un programa de tratamiento psicológico grupal para el tratamiento de los DE en AP, pues pretende promover y facilitar el acceso a tratamientos psicológicos validados empíricamente, a un gran porcentaje de la población. Se trata de un protocolo de tratamiento cuyo propósito es dotar al paciente de los recursos y las herramientas necesarias para gestionar por sí mismo el malestar asociado a situaciones adversas.

El tipo de diseño de caso único empleado para la descripción del presente caso clínico será un diseño ABA (Barlow y Hersen, 1988). Se trata de un diseño de inversión en el que la $A$ indica la primera fase de recogida de datos y evaluación del paciente con el fin de describir la línea base sobre la que se pretende actuar. La $B$ hace referencia a la fase de intervención propiamente dicha, y la última $A$ indica una nueva fase de evaluación y recogida de datos, con el objetivo de comprobar el efecto que ha tenido la intervención en el paciente.

\section{Descripción del caso}

\section{Identificación del paciente}

L. (nombre ficticio), varón de 40 años de edad al inicio de la intervención, casado y sin hijos. Ha estudiado un ciclo de Formación Profesional Básica en Electricidad y Electrónica y actualmente se encuentra el situación de desempleo. Hasta el momento ha trabajado en el sector de la limpieza y en el transporte de mercancías pesadas.

El paciente proviene de una familia de un nivel sociocultural y socioeconómico medio-bajo. Su padre trabajaba en la construcción y su madre era ama de casa.

A nivel familiar, el paciente describe a su padre como una figura de autoridad, distante y poco implicada en la crianza de los hijos, con escasas manifestaciones de afecto. Respecto al perfil de su madre destaca su actitud cariñosa y sobreprotectora, especialmente hacia sus hijos. La define como una persona muy asistencial, con pocas conductas de autocuidado y con un elevado nivel de miedos y preocupaciones, manifestadas diariamente. Con su única hermana, mantiene una relación cordial, aunque un tanto superficial.

Actualmente convive con su pareja, con la que lleva casado 12 años, en una población localizada en el cinturón metropolitano de la ciudad de Valencia. Su mujer ha trabajado esporádicamente en el sector servicios (cuidado de ancianos), y en la actualidad estudia un ciclo medio de Formación Profesional de Técnico en Cuidados Auxiliares de Enfermería.

\section{Análisis del motivo de la intervención}

El paciente acude a su médico de atención primaria (MAP) tras una reciente pérdida de empleo, con un marcado estado ansioso-depresivo, mantenido por un estilo de afrontamiento evitativo. Su trabajo proporcionaba los únicos ingresos familiares a una situación económi- 
ca muy precaria, lo que le ha sumido en un estado de profunda tristeza y culpabilidad.

La sintomatología que presenta, aunque reconoce que ha estado presente en su vida desde hace años, se ha agravado en los últimos meses, lo que interfiere de una manera muy significativa en su día a día, sintiéndose incapaz de manejar la situación, lo que ha precipitado la búsqueda de ayuda profesional.

Concreta el agravamiento de la sintomatología fisiológica, refiriendo una elevada preocupación por la frecuencia de cefaleas, palpitaciones, presión torácica y sensación de mareo e inestabilidad.

\section{Evaluación}

\section{Primer escalón de cribado y derivación. Implementación del PHQ-4}

Su MAP, sensibilizada con la detección de un posible DE, tras observar la sintomatología somática y ansioso-depresiva relatada anteriormente, procede a iniciar la primera fase de un modelo de detección y derivación escalonado de DE en AP en colaboración con el psicólogo clínico de AP, tal y como ha sido descrito en Muñoz-Navarro et al. (2017). En este primer escalón, procede a suministrarle el PHQ-4 para detectar con rapidez y de forma objetiva la presencia de un posible trastorno depresivo o un trastorno de ansiedad generalizada en AP (Cano-Vindel et al., 2018). El paciente puntúa un 4 en los dos ítems correspondientes al GAD-2 y un 5 en los dos ítems del PHQ-2, lo que indica sospecha de presencia de sintomatología ansioso-depresiva. Al observar que rebasa los puntos de corte procede a derivarlo al Psicólogo Clínico por interconsulta.

\section{Segundo escalón de detección. Implementación del PHO}

Tras la derivación del paciente por parte de su MAP y en vista de los resultados del PHQ-4, la Psicóloga Clínica procede a suministrarle un cuestionario más extenso del que se extraen las siguientes puntuaciones:

Somatizaciones: PHQ-15 = 12 (nivel moderado)

Depresión: PHQ-9 = 13 (nivel moderado)

Ansiedad Generalizada: GAD-7 = 19 (nivel severo)

Pánico: PHQ-TP = No

Abuso de alcohol: No

Trastorno de la conducta alimentaria: No

Al cumplir criterios de trastorno de ansiedad generalizada (TAG) a nivel severo, depresión moderada y somatizaciones también a nivel moderado, se confirma la presencia de un DE a nivel moderado, que puede beneficiarse de un tratamiento en el propio centro de AP.

\section{Tercer escalón de confirmación diagnóstica. Entrevista Clínica}

Tras la corrección de las diferentes pruebas de evaluación, la Psicóloga Clínica se dispone a implementar una entrevista semiestructurada para realizar el diagnóstico confirmatorio de la presencia de estos DE con el objetivo de discernir si los cuestionarios de cribado han sido 
lo suficientemente sensibles y específicos, y para descartar un posible trastorno mental más grave. Se utiliza el módulo de TAG de la CIDI (Entrevista Diagnóstica Internacional Compuesta - Composite International Diagnostic Interview) (OMS, 1993), así como el módulo de Episodio de Depresión Mayor de la SCID-I (Entrevista clínica estructurada para los trastornos del eje I del DSM-IV - The Structured Clinical Interview for DSM) (First, Spitzer, Williams y Gibbon, 1999).

\section{Historia del problema}

En la primera consulta realizada por la Psicóloga Clínica, se realiza una primera exploración del paciente que le permite conocer con mayor profundidad la historia de su problema y llegar a un diagnóstico más fiable y específico. L. pertenece a una familia de 4 miembros, siendo el hijo mayor. Su infancia transcurrió en una zona rural, rodeado de su familia extensa. Mantenía una estrecha relación con sus primos, con los que pasaba la mayor parte del tiempo libre. Refiere un suceso en su infancia relacionado con un abuso sexual intrafamiliar, que para el paciente no ha sido motivo de consulta, hasta la fecha, ni conecta con su situación actual.

Se describe como un alumno con bajo rendimiento académico, que tuvo que abandonar los estudios antes de finalizar la enseñanza obligatoria, por necesidad de contribuir económicamente con la familia. Trabajó en el sector de la limpieza y del transporte.

Ha tenido varias relaciones de pareja, siendo las dos últimas, las que refiere como más significativas, por su intensidad y duración. Identifica las relaciones de pareja como el contexto a partir del cual el paciente toma conciencia de sus problemas emocionales.

Describe a su anterior pareja como una persona controladora, manipuladora y muy maniática. Se culpa por no haber escuchado a la gente de su entorno, que le advertía de que se trataba de una mala relación. En la actualidad, el paciente atribuye a esta relación sus problemas económicos, ya que con ella gastó todos sus ahorros, así como su falta de relaciones sociales, pues refiere que ella le aisló de la gente.

Su actual pareja es la persona con la que quiere tener un proyecto de vida en común, a pesar de que en el momento de la entrevista, refiere que su relación de pareja está muy deteriorada, tanto a nivel afectivo como sexual, lo que constituye un importante foco de frustración y culpabilidad. Ambos presentan problemas emocionales, agravados por una insatisfactoria vida sexual y por la situación de desempleo de los dos. L. siente que la responsabilidad económica de la pareja recae sobre él, y se atribuye mayor incompetencia por no conseguir un empleo y unos ingresos estables.

Presenta un estado general de hipolaboriosidad, desorganización y apatía, con dificultades en la toma de decisiones y en el afrontamiento de su situación actual. Además, su vida social es muy reducida e insatisfactoria, pues se aprecian dificultades importantes en las habilidades sociales. 


\section{Análisis funcional}

Figura 1. Análisis funcional de un paciente con un DE.

\begin{tabular}{|c|c|c|}
\hline Antecedentes & Conductas problema & Consecuencias \\
\hline $\begin{array}{l}\text { Situacionales } \\
\text { Por las mañanas, } \\
\text { cuando está solo en casa, } \\
\text { inactivo, "con tiempo } \\
\text { para pensar". } \\
\text { Cuando está con sus }\end{array}$ & $\begin{array}{l}\text { Fisiológicas } \\
\text { Agudización de los } \\
\text { síntomas físicos/ } \\
\text { orgánicos. }\end{array}$ & $\begin{array}{l}\text { Inmediatas } \\
\text { Refuerzo negativo: } \\
\text { Alivio del malestar. } \\
\text { Evasión. } \\
\text { Distracción. }\end{array}$ \\
\hline$\downarrow$ & $\downarrow$ & \\
\hline $\begin{array}{l}\text { Cognitivos } \\
\text { Pensamientos } \\
\text { recurrentes sobre el } \\
\text { pasado: } \\
\text {-Anterior relación de } \\
\text { pareja ("tóxica") } \\
\text {-Gasto de todos sus } \\
\text { ahorros en ella. } \\
\text { Sesgos cognitivos: } \\
\text {-Pensamientos "debería" } \\
\text {-Pensamientos } \\
\text { irracionales, "no valgo } \\
\text { para nada", "nunca hago } \\
\text { nada bien". }\end{array}$ & $\begin{array}{l}\text { Cognitivas } \\
\text { Interpretación } \\
\text { catastrófica de: } \\
\text {-Síntomas físicos. } \\
\text {-Situación económica. } \\
\text {-Relación de pareja. } \\
\text { "Siempre caigo en lo } \\
\text { mismo y no puedo } \\
\text { superarlo". }\end{array}$ & $\begin{array}{l}\qquad \\
\text { A medio plazo } \\
\text { Sentimientos de } \\
\text { culpabilidad y de } \\
\text { fracaso. } \\
\text { Frustración. } \\
\text { Aislamiento social. } \\
\text { Agotamiento. } \\
\text { Vergüenza de sí mismo. } \\
\text { Agravamiento de los } \\
\text { síntomas ansioso- } \\
\text { depresivos. } \\
\text { Retraso en la búsqueda } \\
\text { de empleo. }\end{array}$ \\
\hline$\downarrow$ & relacionadas con la & \\
\hline $\begin{array}{l}\text { Físicos/orgánicos } \\
\text { Dolores/presión en la } \\
\text { cabeza. } \\
\text { Presión torácica. } \\
\text { Mareos, "como si no me } \\
\text { pasara el oxígeno a la } \\
\text { cabeza". } \\
\text { Visión túnel. }\end{array}$ & $\begin{array}{l}\text { búsqueda activa de } \\
\text { empleo y tareas } \\
\text { domésticas. } \\
\text { Uso frecuente de redes } \\
\text { sociales que termina en } \\
\text { visitas a páginas de } \\
\text { pornografía. }\end{array}$ & \\
\hline
\end{tabular}




\section{Diagnóstico}

Tras la revisión de la sintomatología presentada por el paciente y de los datos derivados del cuestionario de evaluación, se establece la hipótesis diagnóstica de Trastorno depresivo persistente (distimia) con ansiedad severa 300.4 (F34.1), según el DSM-5 (APA, 2013), y comorbilidad con somatizaciones. No se observa la presencia de un posible trastorno de la conducta alimentaria, de un trastorno relacionado con sustancias o trastorno adictivo, ni de un trastorno de pánico.

\section{Intervención}

El paciente entra a participar en el proyecto PsicAP, donde se desarrolla un protocolo de tratamiento basado en técnicas cognitivo-conductuales, que incluye un módulo de psicoeducación sobre las emociones y los desórdenes emocionales, entrenamiento en observación y modificación de distorsiones cognitivas, regulación emocional, entrenamiento en relajación muscular progresiva, respiración, imaginación y sugestión, entrenamiento en habilidades conductuales y sociales, exposición a situaciones emocionales y prevención de recaídas (Cordero-Andrés et al., 2017; González-Blanch et al., 2017).

El programa de intervención grupal de PsicAP está fundamentado en el enfoque transdiagnóstico basado en técnicas cognitivo-conductuales (Barlow et al., 2015), que se centra en las comunalidades y semejanzas que presentan y mantienen los distintos DE, enfatizando en los procesos psicológicos que se encuentran a la base de los mismos. El enfoque transdiagnóstico permite realizar mejores diagnósticos y por tanto, comprender y abordar de manera más efectiva la comorbilidad (Belloch, 2012; Farchione et al., 2012).

Los objetivos de este tratamiento están diseñados para que los pacientes aprendan a comprender mejor sus experiencias emocionales, y por tanto a saber gestionar las emociones negativas o desagradables, aprendiendo e incorporando estrategias adaptativas para manejar las experiencias emocionales desagradables.

El programa de tratamiento, diseñado especialmente por Cano-Vindel (Cano-Vindel, 2011b), se aplica en un contexto de AP en formato de terapia grupal. Se compone de 7 sesiones de tratamiento psicológico de hora y media de duración, repartidas a lo largo de 4 meses, desarrolladas en grupos reducidos de 8 a 12 personas. La frecuencia temporal comienza siendo semanal, con una progresiva ampliación del intervalo a medida que avanza el tratamiento (González-Blanch et al., 2017).

\section{Proceso de intervención clínica}

Sesión 1: Presentación y Psicoeducación I (Emociones y Desórdenes emocionales).

El objetivo de la primera sesión de tratamiento consiste en explicar a los pacientes la funcionalidad y el valor adaptativo de las emociones, así como la naturaleza psicosocial y las características principales de los desórdenes emocionales. Se trata de crear un contexto seguro en el que los pacientes participen activamente en su proceso de cambio. 
La sesión comienza con la presentación de la psicóloga clínica, del proyecto PsicAP y de los participantes. Se establecen las normas de la terapia, se reparte el cronograma y se les da acceso a la página web www.desordenesemocionales.es mediante claves personalizadas.

Una vez encuadrado el planteamiento de la terapia, se procede al módulo de psicoeducación, en el que se presentan las emociones básicas y complejas, la funcionalidad social y motivacional de las mismas, y se argumentan las diferencias entre las emociones adaptativas y los trastornos emocionales. Se introducen técnicas de manejo del estrés, entre las que destacan la dieta equilibrada, el descanso, la realización de ejercicio físico, una adecuada organización del tiempo, la solución de problemas y la toma de decisiones, la interpretación de las situaciones, las atribuciones, la autoestima, las relaciones con los demás y el entrenamiento específico en técnicas de control de ansiedad y estrés. Además, se introduce el papel fundamental que juegan los sesgos atencionales e interpretativos en el agravamiento y mantenimiento de las somatizaciones y de la sintomatología ansioso-depresiva.

La sesión finaliza con la introducción a las técnicas de autorregulación emocional, centradas en el manejo de la activación fisiológica. Para ello se enseña la técnica de respiración abdominal o diafragmática.

Tareas para casa:

Lectura de un documento de presentación.

Acceso a la pagina web y biblioterapia.

Realización diaria de la respiración diafragmática.

Cumplimentación de la hoja de autorregistro.

Sesión 2: Psicoeducación II: Estrés y afrontamiento. Relajación.

La segunda sesión, así como todas las siguientes, comienza con un resumen de la sesión anterior y con la revisión de las tareas para casa.

Se comienza realizando preguntas como “¿Qué vimos en la sesión pasada?”, “qué dudas os han surgido?". Se prosigue con la psicoeducación centrada en el modelo de demandas y control (Karasek, 1979) y de esfuerzo y recompensa (Siegrist y Peter, 1996).

Tabla 1.- Ejemplo práctico del modelo de la valoración cognitiva (Lazarus, 1966).

\begin{tabular}{lll}
\hline \multicolumn{1}{c}{ Situación } & \multicolumn{1}{c}{ Valoración cognitiva } & \multicolumn{1}{c}{ Importancia real } \\
\hline Llegar a fin de mes & $\mathbf{9}$ (comer, pagar facturas...) & $\mathbf{9}$ (comer, pagar facturas...) \\
Tareas de la casa & $\mathbf{8}$ (deben estar todas acabadas) & $\mathbf{2}$ (no es para tanto) \\
\multirow{2}{*}{ Empleo } & $\mathbf{9}$ (nunca voy a encontrar empleo, & $\mathbf{6}$ (estoy haciendo todo lo que \\
& no valgo para nada) & puedo) \\
\hline
\end{tabular}


Nuestro paciente ofrece un ejemplo explicando que siempre se encuentra angustiado y preocupado porque no es capaz de establecer una rutina diaria que le facilite realizar una búsqueda activa de empleo y atender las tareas de la casa. En el momento en que su mujer sale de casa y se encuentra solo no consigue iniciar ninguna de las tareas previstas, motivo que le genera niveles elevados de ansiedad que gestiona desadaptativamente mediante el uso desmesurado de las redes sociales. Refiere que esta situación se produce diariamente, por lo que se siente culpable y frustrado.

A continuación se expone el Modelo de la valoración cognitiva de Lazarus (Lazarus, 1966), donde se indica la importancia de la interpretación de la situación, y de la valoración que hacemos de los propios recursos. Se muestra en un ejemplo del paciente cómo a veces se valora una situación poco estresante como muy estresante. Por tanto, se hace énfasis de nuevo en que la reacción ante un suceso depende en gran medida, de lo que se piense y de lo que se haga.

Finalmente, se repasa la respiración diafragmática, revisando las tareas para casa y realizando una práctica grupal, y se introduce, como estrategia de autorregulación emocional, la relajación muscular progresiva (Jacobson, 1929).

L. refiere que la respiración diafragmática le está ayudando mucho para aprender a gestionar la emoción en momentos de elevada intensidad, y que la está poniendo en práctica diariamente, tal y como pauta el protocolo de tratamiento.

Tareas para casa:

Práctica y registro diario de la respiración diafragmática.

Práctica y registro de la relajación muscular progresiva (formato CD).

Lectura del documento de introducción a la terapia cognitiva.

Acceso a la página web.

\section{Sesión 3: Reestructuración cognitiva I.}

El objetivo de la tercera sesión es continuar afianzando las estrategias de autorregulación emocional e introducir la realización de actividades agradables y ejercicio físico o deporte. Además, se introduce la reestructuración cognitiva con el fin de que los pacientes detecten sus propios estilos desadaptativos de pensamiento, así como los sesgos cognitivos más frecuentes (sesgo atencional y sesgo interpretativo), que les están causando un elevado malestar emocional. Para ello, también se hace hincapié en el concepto de pensamiento irracional y pensamiento racional.

Finalmente, se presenta el modelo de expectativas y la profecía autocumplida (Rosenthal, 1974) y se introduce la teoría atribucional (Weiner, 1988).

Se aplica la técnica de la flecha descendente para que el paciente observe qué tipo de sesgos cognitivos y pensamientos irracionales puede estar utilizando. 
Tabla 2.- Ejemplo práctico de la técnica de la flecha descendente.

\section{¿Qué hechos de mi vida me generan ansiedad o me afectan?}

No he hecho las tareas propuestas de búsqueda de empleo y domésticas.

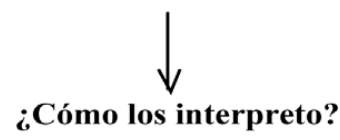

No he hecho nada de lo que me había propuesto.

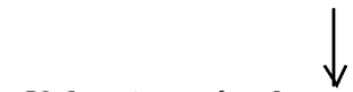

¿Y de esto, qué es lo que realmente me afecta?

Debería atender mis responsabilidades como lo hace todo el mundo.

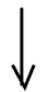

¿Y de esto, qué es lo que realmente me afecta?

Que cuando llegue mi mujer a casa volveremos a tener un conflicto.<smiles>C1CCCCC1</smiles>

¿Y de esto, qué es lo que realmente me afecta?

Que nunca soy capaz de cumplir con mis obligaciones.

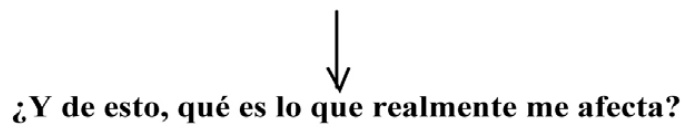

Que no valgo para nada porque siempre caigo en lo mismo y no puedo superarlo.

Tareas para casa:

Práctica y registro diario de la respiración diafragmática.

Práctica y registro de la relajación muscular progresiva (CD).

Práctica y registro de las actividades agradables o ejercicio físico.

Autorregistro: Situación/emoción/conducta/consecuencias.

Lectura del documento de distorsiones de pensamiento y sesgos cognitivos.

Acceso a la página web. 


\section{Sesión 4: Reestructuración cognitiva II.}

La cuarta sesión continúa afianzando la reestructuración cognitiva con el objetivo de modificar los estilos desadaptativos de pensamiento, introduciendo el uso de autoinstrucciones positivas y la ideación de un experimentos conductual.

En primer lugar se realiza un resumen de la sesión anterior y se refuerzan las estrategias de autorregulación emocional: la respiración diafragmática, la relajación muscular progresiva y la realización de actividades agradables y ejercicio físico.

El paciente acude a la sesión muy contento y motivado, pues está muy implicado en el programa y está obteniendo muy buenos resultados hasta el momento. Utiliza diariamente la respiración diafragmática para gestionar las situaciones que le generan una elevada intensidad emocional, y ha introducido la relajación muscular progresiva, que refiere que también le ayuda a relajarse y a ser más consciente de su cuerpo, ya que ha aprendido a detectar las situaciones diarias en las que está tenso, destensando los músculos y adoptando una postura más cómoda y menos rígida.

Tabla 2.- Aplicación práctica del Modelo de autoinstrucciones positivas (Meichenbaum, 1985).

\begin{tabular}{|c|c|c|c|c|}
\hline Situación & Pensamiento & Consecuencia & $\begin{array}{c}\text { Pensamiento } \\
\text { alternativo }\end{array}$ & Consecuencia \\
\hline $\begin{array}{l}\text { Llegar a } \\
\text { fin de mes }\end{array}$ & $\begin{array}{l}\text { No podré pagar } \\
\text { el alquiler ni } \\
\text { los recibos de la } \\
\text { casa. }\end{array}$ & $\begin{array}{l}\text { La cuenta se queda- } \\
\text { rá en números rojos } \\
\text { y el banco me dará } \\
\text { problemas. }\end{array}$ & $\begin{array}{l}\text { Si gasto lo nece- } \\
\text { sario podré repar- } \\
\text { tir el dinero para } \\
\text { lo necesario }\end{array}$ & $\begin{array}{l}\text { No tendré pro- } \\
\text { blemas con el } \\
\text { banco. }\end{array}$ \\
\hline $\begin{array}{l}\text { Tareas de } \\
\text { la casa }\end{array}$ & $\begin{array}{l}\text { Debería tener } \\
\text { la casa limpia y } \\
\text { ordenada todos } \\
\text { los días. }\end{array}$ & $\begin{array}{l}\text { Me desborda y me } \\
\text { frusto porque no } \\
\text { puedo con todo. }\end{array}$ & $\begin{array}{l}\text { Voy a marcarme } \\
\text { objetivos más } \\
\text { pequeños y al- } \\
\text { canzables. }\end{array}$ & $\begin{array}{l}\text { Me sentiré sa- } \\
\text { tisfecho de los } \\
\text { logros consegui- } \\
\text { dos. }\end{array}$ \\
\hline Empleo & $\begin{array}{l}\text { Ya debería haber } \\
\text { encontrado un } \\
\text { empleo. }\end{array}$ & $\begin{array}{l}\text { Me genera mucha } \\
\text { ansiedad llevar va- } \\
\text { rios meses en paro. }\end{array}$ & $\begin{array}{l}\text { No es fácil en- } \\
\text { contrar empleo } \\
\text { pero me voy a } \\
\text { esforzar. }\end{array}$ & $\begin{array}{l}\text { Me siento mas } \\
\text { tranquilo y puedo } \\
\text { seguir buscando } \\
\text { empleo sin tanta } \\
\text { presión. }\end{array}$ \\
\hline
\end{tabular}

Por otro lado, refiere que se ha establecido una rutina en la que ha incorporado la realización de actividades agradables y actividad física, entre las que destacan las excursiones por el campo, unas veces solo y otras veces con su mujer, y los paseos con sus mascotas. Ha retomado la escritura, una actividad muy placentera que además resulta muy terapéutica, pues comenta que escribir le sirve de ventilación emocional, ya que le ayuda a ser consciente y procesar de una forma más adaptativa las situaciones estresantes. 
A continuación, se hace un repaso grupal de los contenidos tratados en la sesión anterior sobre la terapia cognitiva, haciendo hincapié en los sesgos atencionales e interpretativos, junto con la identificación de los sesgos de nuestro paciente. L. afirma que se ha dado cuenta de que tiene una gran cantidad de pensamientos irracionales. Identifica pensamientos absolutistas (siempre caigo en lo mismo y no puedo superarlo, o, nunca me sale nada bien), pensamientos rígidos (no valgo para nada), los que adivinan el futuro (seguro que me rechazan), y aquellos que expresan exigencias (debería estar todo controlado, o, debería hacerlo bien a la primera). Esto le ha servido para ser consciente del papel que juegan sus propios pensamientos en el incremento del malestar emocional que siente.

Se presentan tres tareas fundamentales que forman parte de la reestructuración cognitiva. La primera consiste en identificar los pensamientos que pueden generar ansiedad, la segunda en identificar los errores cometidos en estos procesos, y la tercera en cambiar los errores cometidos por procesos más adaptativos y pensamientos más racionales.

La sesión continúa con la presentación del modelo de autoinstrucciones positivas (Meichenbaum, 1977), con el objetivo de promover la generación de pensamientos alternativos.

Una vez identificados los pensamientos y su funcionalidad en la conducta del paciente, se entrena la generación de pensamientos alternativos, que provocarán respuestas más adaptativas en la vida del paciente.

A continuación se presenta un ejemplo de dicha técnica trabajada con el paciente:

Finalmente, se introduce la ideación de un experimento conductual a poner en práctica ante los pensamientos irracionales, animando al grupo a asignarse un experimento como tarea para casa. Ello contribuirá a desconformar las creencias irracionales y facilitará la eliminación de posibles conductas de seguridad, que puedan estar manteniendo el problema.

El experimento conductual que se propone el paciente es el siguiente: "Voy a tener el ordenador apagado desde que me levante hasta las 10 de la mañana, mientras ordeno la habitación y recojo el desayuno".

Tareas para casa:

Práctica y registro diario de la respiración diafragmática.

Práctica y registro de la relajación muscular progresiva (CD).

Práctica y registro de las actividades agradables o ejercicio físico.

Práctica y registro de las actividades de reestructuración cognitiva.

Realización del experimento conductual.

Acceso a la página web.

Sesión 5 y 6: Reestructuración cognitiva III y solución de problemas.

Ambas sesiones comienzan con el repaso del contenido de la sesión anterior con las preguntas “¿qué vimos en la sesión pasada?”, “¿qué dudas os han surgido?”. A continuación se refuerzan las estrategias de autorregulación emocional, se revisan los registros y el experimento conductual. 
El paciente comenta que realizó con éxito el experimento conductual propuesto. Fue capaz de conseguir el objetivo y no encender el ordenador a primera hora de la mañana, dedicando ese intervalo de tiempo a la realización de las tareas del hogar. Se sintió muy satisfecho y orgulloso de conseguirlo, sentimiento que aumentaba conforme pasaban los días.

En las sesiones 5 y 6 se continúa trabajando y afianzando la reestructuración cognitiva, añadiendo el entrenamiento en la técnica de resolución de problemas (D’Zurilla y Goldfried, 1971). Además, se trabajan técnicas psicológicas como la autoobservación, el control de estímulos, el entrenamiento conductual reforzado, la exposición sin conductas de seguridad y el entrenamiento en habilidades de afrontamiento.

En la sesión se revisan ejemplos de sucesos emocionales vividos recientemente, reforzando los logros que hayan alcanzado los pacientes en la forma de interpretar la situación y de gestionarla, apuntando aquellas áreas que requieran seguir trabajando y poniendo más tareas para casa para que continúen exponiéndose a situaciones que generan malestar, ensayando, practicando y corrigiendo errores progresivamente.

Tareas para casa:

Práctica y registro diario de la respiración diafragmática.

Práctica y registro de la relajación muscular progresiva $(\mathrm{CD})$.

Práctica y registro de las actividades agradables o ejercicio físico.

Autoaplicación y registro de la reestructuración cognitiva.

Autoaplicación y registro de experimentos conductuales.

Acceso a la página web.

Sesión 7: Prevención de recaídas y cierre.

La séptima y última sesión de tratamiento, consiste en repasar y reforzar las estrategias cognitivo-conductuales y de autorregulación emocional adquiridas durante todo el programa, y aprender técnicas de prevención con el objetivo de reducir la posibilidad de experimentar recaídas.

Para ello, se comienza con el resumen de la sesión anterior y la revisión de las tareas para casa, y se procede a resaltar y animar a los participantes a mantener el uso de aquellas estrategias que les han resultado útiles. Se enfatiza la diferencia entre caída y recaída, y de sensación de fracaso frente a oportunidad de aprendizaje. De esta manera, se señala que las recaídas no significan volver al principio, ni una pérdida total de las ganancias terapéuticas, ni siquiera es un fracaso, sino que se puede interpretar como un nuevo reto, como una oportunidad para aprender y consolidar lo ya aprendido. A continuación, se identifican las áreas o situaciones de riesgo que perciba cada paciente, animándoles a que busquen y pongan en práctica estrategias para gestionarlas con éxito.

L. identifica como situaciones potenciales de riesgo quedarse solo en casa y pasar tiempo inactivo, tras haber tenido algún conflicto con su mujer. 


\section{Resultados}

Tras la finalización de las 7 sesiones de tratamiento grupal, se observan mejorías significativas en el paciente. Refiere una notable mejoría de su estado de ánimo, pues ya no se siente tan ansioso ni tan deprimido. Ha aprendido a detectar los pensamientos irracionales y los sesgos cognitivos que provocaban un aumento de su malestar, siendo ahora capaz de gestionar las situaciones desagradables y estresantes, haciendo uso de las estrategias de autorregulación aprendidas. Asimismo, haber encontrado trabajo (durante el periodo de tratamiento), le ha ayudado a estructurar su vida, estableciendo una rutina y contribuyendo a mejorar su difícil situación económica. Además, en el momento de finalizar el programa, se observa que el paciente es capaz de verbalizar sus dificultades con mayor asertividad y precisión, y que ha avanzado notablemente en la externalización del síntoma.

Estos resultados cualitativos se apoyan en los resultados derivados de la evaluación posttratamiento:

Somatizaciones: PHQ-15 = 2 (inexistente)

Depresión: PHQ-9 = 5 (nivel leve)

Ansiedad Generalizada: GAD-7 = 5 (nivel leve)

Pánico: PHQ-TP = No

Abuso de alcohol: No

Trastorno de la conducta alimentaria: No

Estas puntuaciones indican una reducción significativa de la sintomatología clínica ansiosodepresiva y de las somatizaciones. Además, L. refiere que el nivel de interferencia de su sintomatología actual es leve, por lo que no le supone ninguna dificultad en el desarrollo de su vida cotidiana, en las relaciones sociales, en las relaciones familiares o en el desempeño de su trabajo.

\section{Seguimiento}

Se realizaron sesiones de seguimiento con el paciente con un intervalo de 3, 6 y 12 meses. Los resultados obtenidos en los cuestionarios de seguimiento, informaron del mantenimiento y consolidación de los logros terapéuticos conseguidos durante el tratamiento (ver figura 3).

En estas sesiones se evidenció un cambio importante en la interpretación de las situaciones estresantes, un aumento de su flexibilidad cognitiva y una mayor habilidad de resolución de problemas. Se mostraba más enérgico y alegre, y comunicaba ilusión por actividades compartidas con su pareja.

Estos resultados fueron, asimismo, corroborados por el paciente, que destacó su satisfacción por haber participado en la terapia grupal, incidiendo en los beneficios que le había reportado en su vida diaria.

A partir de estos resultados, se considera que el tratamiento psicológico aplicado en formato grupal, se ha mostrado eficaz para el abordaje de los DE en AP, ya que ha proporcionado mejorías significativas duraderas en la calidad de vida y en la capacidad de adaptación del paciente. 
Figura 3. Representación gráfica de los resultados obtenidos por un paciente con un DE en somatizaciones (PHQ-15), depresión (PHQ-9) y ansiedad (GAD-7) en la fase previa al tratamiento (PRE), en la fase posterior al tratamiento (POST) y durante el seguimiento: a los 3 meses de finalizar el tratamiento $(3 \mathrm{M})$, tras 6 meses $(6 \mathrm{M})$ y tras 12 meses $(12 \mathrm{M})$.

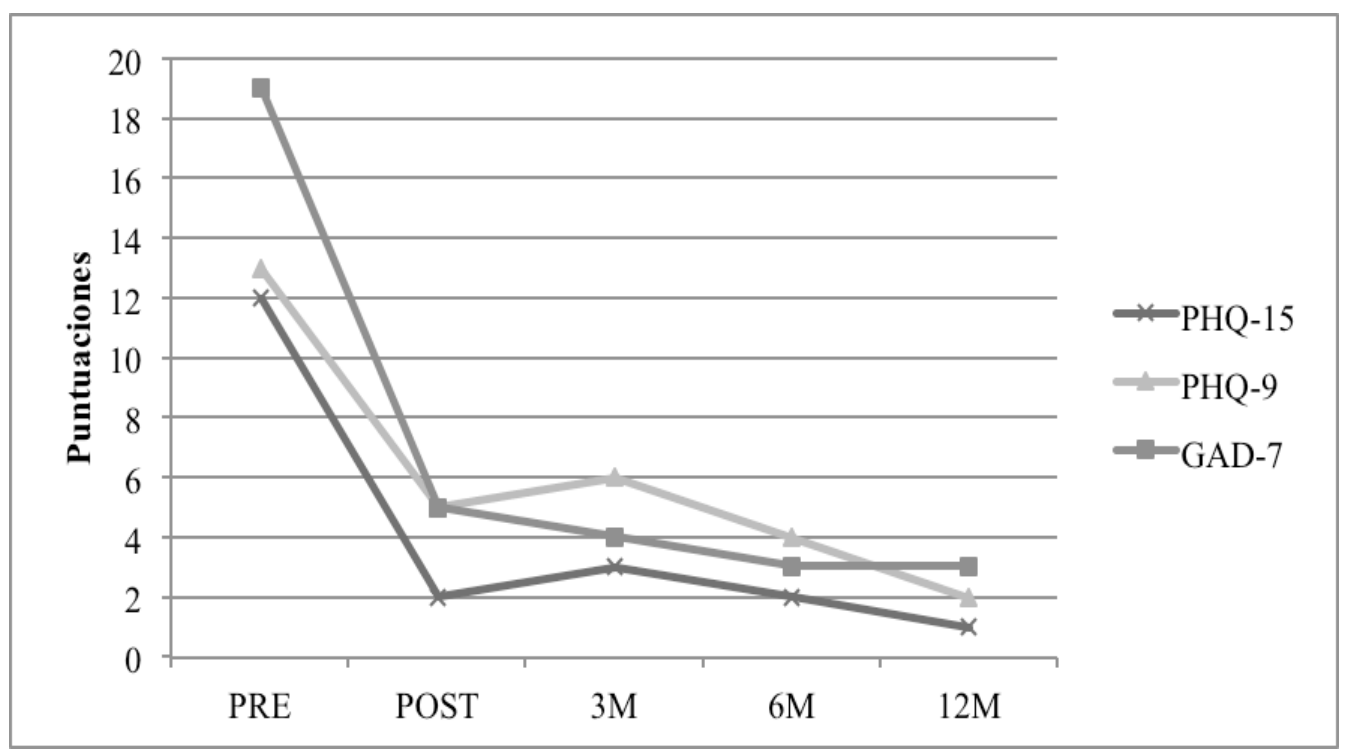

\section{Discusión y conclusiones}

El objetivo del presente artículo era la presentación detallada de un caso clínico de un DE atendido en AP mediante un protocolo de intervención basado en el enfoque transdiagnóstico. Para ello se ha descrito el caso, el motivo de la intervención, el cribado del MAP y la evaluación y diagnóstico del paciente por parte de la Psicóloga Clínica. Además, se ha descrito el proyecto PsicAP y las siete sesiones del programa de intervención psicológica grupal que se aplicó al paciente, presentando finalmente los resultados.

El enfoque transdiagnóstico permite aunar los factores comunes que comparten distintos trastornos para así diseñar programas de tratamiento que abarquen un rango amplio de psicopatologías, de manera que estos tratamientos transdiagnósticos resulten especialmente eficaces en su implementación de forma grupal. Este es uno de los aspectos fundamentales que dotan a estos programas de las características idóneas para su puesta en marcha en el contexto de AP, es decir, un contexto de gran demanda asistencial y escasos recursos (Belloch, 2012).

Además, se trata de un protocolo que aborda los $\mathrm{DE}$, que se encuentran entre los problemas mentales más frecuentes con prevalencia vital de alrededor del $25 \%$ y elevadas tasas de comorbilidad. Está basado en técnicas cognitivo-conductuales que cuentan con gran evidencia 
científica para el abordaje de los DE, por lo que mejora uno de los principales problemas a los que se enfrentan los profesionales de la salud mental: la accesibilidad a las intervenciones psicológicas basadas en la evidencia (Barlow, 2015).

Al aplicarse en AP, el primer nivel asistencial que recibe numerosas consultas relacionadas con trastornos emocionales, resulta especialmente eficiente ya que permite intervenir desde las primeras fases de los trastornos, evitando así en muchas ocasiones la cronificación de los mismos, y beneficiando indirectamente a los servicios de atención especializada (GonzálezBlanch, et al., 2017).

El presente artículo, a pesar de exponer un caso único, puede servir de muestra de la eficacia de este breve y sencillo protocolo de intervención, tal y como están mostrando los resultados preliminares de la investigación. Se evidencian cambios significativos en los pacientes con DE tras su participación activa en el programa, manteniéndose estos cambios estables a largo plazo.

\section{Referencias}

American Psychiatric Association. (2013). Manual Diagnóstico y Estadístico de los Trastornos Mentales (DSM $5^{\oplus)} 5^{\circ}$. Ed. Arlington, VA, Asociación Americana de Psiquiatría (or. ingl., 2013).

Barlow, D. H., Farchione, T. J., Fairholme, C. P., Ellard, K. K., Boisseau, C. L., Allen, L. B. y Ehrenreich-May, J. (2015). Protocolo unificado para el tratamiento transdiagnóstico de los trastornos emocionales. Madrid: Alianza Editorial.

Barlow, D. H. y Hersen, M. (1988). Diseños experimentales de caso único. Barcelona: Martínez Roca.

Belloch, A. (2012). Propuestas para un enfoque transdiagnóstico de los trastornos mentales y del comportamiento: evidencia, utilidad y limitaciones. Revista de Psicopatología y Psicología Clínica, 17(3), 295-311.

Bortolotti, B., Menchetti, M., Bellini, F., Montaguti, M. B., y Berardi, D. (2008). Psychological interventions for major depression in primary care: a meta-analytic review of randomized controlled trials. General Hospital Psychiatry, 30, 293-302.

Bullis, J. R., Sauer-Zavala, S., Bentley, K. H., Thompson-Hollands, J., Carl, J. R. y Barlow, D. H. (2015). The unified protocol for transdiagnostic treatment of emotional disorders: Preliminary exploration of effectiveness for group delivery. Behavior Modification, 39, 295-321.

Cano-Vindel, A. (2011a). Los desórdenes emocionales en Atención Primaria. Ansiedad y Estrés, 17(1), 75-97.

Cano-Vindel, A. (2011b). Bases teóricas y apoyo empírico de la intervención psicológica sobre los desórdenes emocionales en Atención Primaria. Una actualización. Ansiedad y Estrés, 17(2-3), 157-184.

Cano-Vindel, A., Muñoz-Navarro, R., Medrano, L. A., Ruiz-Rodríguez, P., González-Blanch, C., Castillo, M. D. G., ... y PsicAP Research Group. (2018). A computerized version of the Patient Health Questionnaire-4 as an ultra-brief screening tool to detect emotional disorders in primary care. Journal of Affective Disorders, 234(1), 247-255.

Cape, J., Whittington, C., Busze- wicz, M., Wallace, P. y Underwood, L. (2010). Brief psychological therapies for anxiety and depression in primary care: meta-analysis and meta-regression. BMC Medicine, 8, 38.

Chu, B. C., Crocco, S. T., Esseling, P., Areizaga, M. J., Lindner, A. M., \& Skriner, L. C. (2016). Transdiagnostic group behavior al activation and exposure therapy for youth anxiety and de- pression: Initial randomized controlled trial. $B e-$ haviour Research and Therapy, 76,65-75.

Cordero-Andrés, P., González-Blanch, C., Umaran-Alfageme, 0., Muñoz-Navarro, R., Ruíz-Rodríguez, P., Adrián-Medrano, L., Hernándezz-de Hita, F., Pérez-Poo, T., Cano-Vindel, A. y Grupo de Investigación PsicAP. (2017). Tratamiento 
psicológico de los trastornos emocionales en atención primaria: fundamentos teóricos y empíricos del estudio PsicAP. Ansiedad y Estrés, 23, 91-98.

D'Zurilla, T. J. y Goldfried, M. R. (1971). Problem solving and behavior modification. Journal of Abnormal Psychology, $78,107$.

Espejo, E. P., Castriotta, N., Bessonov, D., Kawamura, M., Wer- dowatz, E. A. y Ayers, C. R. (2016). A pilot study of transdiagnostic group cognitive-behavioral therapy for anxiety in a veteran sample. Psychological Services, 13, 162.

Farchione, T. J., Fairholme, C. P., Ellard, K. K., Boisseau, C. L., Thompson-Hollands, J., Carl, J. R. y ... Barlow, D. H. (2012). Unified protocol for transdiagnostic treatment of emotional disorders: A randomized controlled trial. Behaviour Therapy, 43, 666-678.

First, M. B., Spitzer, R. L., Williams, J. B. W. y Gibbon, M. (1999). Entrevista Clínica Estructurada para los Trastornos del Eje I del DSM-IV, Versión Clínica (SCID-I-VC). Barcelona: Masson.

García-Escalera, J., Chorot, P., Valiente, R. M., Reales, J. M. y Sandín, B. (2016). Efficacy of transdiagnostic cognitivebehavioral therapy for anxiety and depression in adults, children and adolescents: A meta-analysis. Revista de Psicopatología y Psicología Clínica, 21, 147-175.

González-Blanch, C., Umaran-Alfageme, 0., Cordero-Andrés, P., Muñoz-Navarro, R., Ruíz-Rodríguez, P., Adrián-Medrano, L., Ruíz-Torres, M., Dongil-Collado, E., Cano-Vindel, A. y Grupo de Investigación PsicAP. (2017). Tratamiento psicológico de los trastornos emocionales en Atención Primaria: el manual de tratamiento transdiagnóstico del estudio PsicAP. Ansiedad y Estrés, 24, 1-11.

Haro, J. M., Palacín, C., Vilagut, G., Martínez, M., Bernal, M., Luque, I. y Grupo ESEMeD-España. (2006). Prevalencia de los trastornos mentales y factores asociados: resultados del estudio ESEMeD-España. Medicina Clínica (Barc), 126(12), 445-451.

Huntley, A., Araya, R. y Salisbury, C. (2012). Group psychological therapies for depression in the community: Systematic review and meta-analysis. The British Journal of Psychiatry, 200, 184-190.

Ito, M., Horikoshi, M., Kato, N., Oe, Y., Fujisato, H., Nakajima, S., Kanie, A., Miyamae, M.,Takebayashi Y., Horita, R.,Usuki, M., Nakagawa, A. y Ono Y. (2016). Transdiagnostic and transcul- tural: Pilot study of unified protocol for depressive and anxiety disorders in Japan. Behavior therapy, 47, 416-430.

Jacobson, E. (1929). Progressive relaxation. Oxford, Inlgaterra: University of Chicago Press.

Karasek, R. A. (1979). Job demands, job decision latitude, and mental strain: Implications for job redesign. Administrative Science Quarterly, 24, 285-308.

Kovess-Masfety, V., Alonso, J., Brugha, T., Angermeyer, M., Haro, J., Sevilla-Dedieu, C. y ESEMeD/MHEDEA 2000 Investigators. (2007). Differences in lifetime use of services for mental health problems in six European countries. Psychiatric Services, 58, 213-220.

Lazarus, R. S. (1966). Psychological stress and the coping process. Nueva York: McGraw- Hill.

Meichenbaum, D. (1977). Cognitive behaviour modification. Scandinavian Journal of Behaviour Therapy, 6, 185192.

Mullin, A., Dear, B. F., Karin, E., Wootton, B. M., Staples, L. G., Johnston, L. y Titov, N. (2015). The Uni Wellbeing course: A randomized controlled trial of a transdiagnostic internet-delivered cognitive behavior al therapy (CBT) programme for university students with symptoms of anxiety and depression. Internet Interventions, 2, 128136.

Muñoz-Navarro, R., Cano-Vindel, A., Ruiz-Rodríguez, P., Medrano, L. A., González-Blanch, C., Moriana, J. A., ... y DongilCollado, E. (2017). Modelo jerárquico de diagnóstico y derivación de los trastornos mentales comunes en centros de atención primaria. Una propuesta a partir del ensayo clínico PsicAP. Ansiedad y Estrés, 23(4)، 124-129.

Newby, J. M., McKinnon, A., Kuyken, W., Gilbody, S. y Dalgleish, T. (2015). Systematic review and meta-analysis of transdiagnostic psychological treatments for anxiety and depressive disorders in adulthood. Clinical Psychological Review, 40, 91-110. 
Organización Mundial de la Salud (1993). Composite International Diagnostic Interview, Versión 2.1. Génova: OMS.

Organización Mundial de la Salud (2017). Trastornos mentales. Recuperado de: http://www. who.int/mediacentre/ factsheets/fs396/es/

Osma, J., Castellano, C., Crespo, E. y García-Palacios, A. (2015). The Unified Protocol for transdiagnostic treatment of emotional disorders in group format in a spanish public mental health setting. Behavioral Psychology, 23, 447-466.

Richards, D. A. y Suckling, R. (2009). Improving access to psychological therapies: phase IV prospective cohort study. British Journal of Clinical Psychology, 48, 377-396.

Rosenthal, R. (1974). On the social psychology of self-fulfilling prophecy: Further evidence for Pygmalion effects and their mediating mechanisms. Nueva York: Holt, Rinehart y Winston.

Siegrist, J. y Peter, R. (1996). Measuring effort-reward imbalance at work: Guidelines. Düsseldorf: Heinrich Heine University.

Weiner, B. (1988). Attribution theory and attributional therapy: Some theoretical observations and suggestions. British Journal of Clinical Psychology, 27, 99-104. 